\title{
CENTRALIZER NEAR-RINGS THAT ARE ENDOMORPHISM RINGS
}

\author{
CARLTON J. MAXSON AND KIRBY C. SMITH
}

\begin{abstract}
For a finite ring $\boldsymbol{R}$ with identity and a finite unital $\boldsymbol{R}$-module $\boldsymbol{V}$ the set $C(R ; V)=\{f: V \rightarrow V \mid f(\alpha v)=\alpha f(v)$ for all $\alpha \in R, v \in V\}$ is the centralizer near-ring determined by $R$ and $V$. Those rings $R$ such that $C(R ; V)$ is a ring for every $R$-module $V$ are characterized. Conditions are given under which $C(R ; V)$ is a semisimple ring. It is shown that if $C(R ; V)$ is a semisimple ring then $C(R ; V)=$ $\operatorname{End}_{R}(V)$.
\end{abstract}

1. Preliminaries. Let $G$ be a group and $\Gamma$ a semigroup of endomorphisms of $G$. Then $C(\Gamma ; G)=\{f: G \rightarrow G \mid f(0)=0$ and $f(\gamma a)=\gamma f(a)$ for all $\gamma \in \Gamma, a \in G\}$ is a near-ring under the operations of function addition and function composition, and is called the centralizer near-ring determined by $\Gamma$ and $G$. Moreover, every near-ring with identity arises in this manner [6, p. 50]. It has been shown by Betsch [1] that $N$ is a finite simple near-ring with identity if and only if there exists a finite group $G$ and a fixed point free group of automorphisms $\Gamma$ of $G$ such that $N \cong C(\Gamma ; G)$. The structure of $C(\Gamma ; G)$ for various $G$ 's and $\Gamma$ 's has been investigated in [2], [3] and [4].

Throughout this paper $R$ will denote a finite ring with 1 and $V$ a finite unital $R$-module. The corresponding centralizer near-ring is $C(R ; V)=\{f: V \rightarrow V \mid f(r v)$ $=r f(v)$ for all $r \in R, v \in V\}$. In dealing with $C(R ; V)$ we may assume, without loss of generality, that $V$ is a faithful $R$-module, for we have $C(R ; V)=C(\bar{R} ; V)$ where $V$ is a faithful $\bar{R}$-module, $\bar{R}=R / \operatorname{Ann}(V)$.

In [5] we showed that if $R$ is a finite simple ring then $C(R ; V)$ is a simple near-ring. This result is used to obtain the following generalization.

Proposition. Let $R$ be a finite semisimple ring and let $V$ be a finite $R$-module. Then $C(R ; V)$ is a semisimple near-ring.

Proof. We have $R=S_{1} \oplus \cdots \oplus S_{t}$ where each $S_{i}$ is a simple ring. Let $e_{i}$ denote the identity of $S_{i}$. If $V_{i}=\left\{v \in V \mid e_{i} v=v\right\}$ then $V=V_{1} \oplus \cdots \oplus V_{t}$ and $f\left(V_{i}\right) \subseteq V_{i}$ for each $f \in C(R ; V)$. Further, if $f_{i}$ denotes the restriction of $f$ to $V_{i}$ then the map $\phi: C(R ; V) \rightarrow C\left(S_{1} ; V_{1}\right) \oplus \cdots \oplus C\left(S_{t} ; V_{t}\right)$ given by $\phi(f)=$ $\left\langle f_{1}, \ldots, f_{t}\right\rangle$ is a near-ring homomorphism. The map is onto, for if $\left\langle f_{1}, \ldots, f_{t}\right\rangle$ is in $C\left(S_{1} ; V_{1}\right) \oplus \cdots \oplus C\left(S_{t} ; V_{t}\right)$ extend each $f_{i}$ to all of $V$ by $\bar{f}_{i}\left(v_{1}+\cdots+v_{t}\right)=$ $f_{i}\left(v_{i}\right)$. Then $f=\sum \bar{f}_{i}$ is an element of $C(R ; V)$ such that $\phi(f)=\left\langle f_{1}, \ldots, f_{t}\right\rangle$. To

Received by the editors July 26, 1979 and, in revised form, October 30, 1979.

AMS (MOS) subject classifications (1970). Primary 16A76, 16A44; Secondary 16A42, 16A48.

Key words and phrases. Centralizers, near-rings, semisimple rings. 
show that $\Phi$ is one-to-one we note that $e_{i} f\left(v_{1}+\cdots+v_{t}\right)=f\left(e_{i} v_{i}\right)=f\left(v_{i}\right), i=$ $1, \ldots, t$, implies $f\left(v_{1}+\cdots+v_{t}\right)=f\left(v_{1}\right)+\cdots+f\left(v_{t}\right)=f_{1}\left(v_{1}\right)+\cdots+f_{t}\left(v_{t}\right)$. Hence if $\phi(f)=0$ then $f=0$. Therefore $\phi$ is an isomorphism and from Theorem 1 of [5] each $C\left(S_{i} ; V_{i}\right)$ is a simple near-ring.

A type of converse to the proposition is also true. If $C(R ; V)$ is a semisimple near-ring for every $R$-module $V$ then in particular $C(R ; R)$ is semisimple. But $C(R ; R)$ is anti-isomorphic to $R$ so $R$ is a semisimple ring.

Again using Theorem 1 of [5] if $R \cdot=S_{1} \oplus \cdots \oplus S_{t}, S_{i}$ simple and not a field, or $S_{i}$ is a field and $\operatorname{dim}_{S_{i}}\left(V_{i}\right)=1$, we have $C(R ; V)$ is a semisimple ring. Moreover, in this setting, $C(R ; V)=\operatorname{End}_{R}(V)$. (See proof of Theorem 1 of [5].)

It is the goal of this paper to consider the following questions which arise naturally from the above remarks.

A. Which finite rings $R$ have the property that $C(R ; V)$ is a ring for every $R$-module $V$ ?

B. If $C(R ; V)$ is a semisimple ring when is $C(R ; V)=\operatorname{End}_{R}(V)$ ?

C. Which semisimple near-rings have the form $C(R ; V)$ for some pair $(R, V)$ ?

In the next section we answer question $\mathrm{A}$. In $\S 3$ we show that if $C(R ; V)$ is a semisimple ring then one always has $C(R ; V)=\operatorname{End}_{R}(V)$. Moreover if $C(R ; V)$ is semisimple then information about the structure of the simple components is obtained, giving a partial answer to question $\mathrm{C}$.

2. Strongly noncommutative rings. In this section we characterize those rings $R$ such that $C(R ; V)$ is a ring for every $V$. Recall that if $R$ is a finite ring with identity then $R=T+M$ where $T \cap M=(0), M$ is a subgroup of $\operatorname{rad} R$ and $T=T_{1}$ $\oplus \cdots \oplus T_{t}, T_{i}$ a complete $n_{i} \times n_{i}$ matrix ring over a local $\operatorname{ring} L_{i}$ with $T / \operatorname{rad} T \cong$ $R / \operatorname{rad} R[7$, p. 162]. Moreover there exist mutually orthogonal idempotents $e_{1}, \ldots, e_{t}$ in $R$ such that $1=e_{1}+\cdots+e_{t}$ and $T_{i}=e_{i} R e_{i}$ for each $i$. Also $R / \operatorname{rad} R=S_{1} \oplus \cdots \oplus S_{t}$ where each $S_{i}$ is an $n_{i} \times n_{i}$ simple matrix ring and $T_{i}$ is mapped onto $S_{i}$ under the natural homomorphism $R \rightarrow R / \operatorname{rad} R$ (see [7, p. 162-163]). We say $R$ is strongly noncommutative if $n_{i}>1$ for $i=1,2, \ldots, t$.

THEOREM 2.1. For a finite ring $R$ with identity the following are equivalent:

(i) $C(R ; V)$ is a ring for every faithful $R$-module $V$;

(ii) $C(R ; V)=\operatorname{End}_{R}(V)$ for every faithful $R$-module $V$;

(iii) $R$ is strongly noncommutative.

Proof. Since (ii) implies (i) is clear it remains to show (iii) implies (ii) and (i) implies (iii).

Suppose $R$ is strongly noncommutative where, as above, $R=T+M, T=T_{1}$ $\oplus \cdots \oplus T_{t}$ with each $T_{i}$ an $n_{i} \times n_{i}$ matrix ring over a local ring $L_{i}$ and $n_{i}>1$ for each $i$. If $V$ is a faithful $R$-module then $V$ is a faithful, unital $T$-module and $C(R ; V) \subseteq C(T ; V)$. Thus it suffices to show that for each $f \in C(T ; V)$ and for each $v, w \in V, f(v+w)=f(v)+f(w)$. To this end let $e_{i}$ be the identity for $T_{i}$; then $V=V_{1} \oplus \cdots \oplus V_{t}$ where $V_{i}=e_{i} V$. We have $f\left(v_{1}+\cdots+v_{t}\right)=f\left(v_{1}\right)$ $+\cdots+f\left(v_{t}\right), v_{i} \in V_{i}$, so it suffices to show $f\left(v_{i}^{1}+v_{i}^{2}\right)=f\left(v_{i}^{1}\right)+f\left(v_{i}^{2}\right)$ for every $v_{i}^{1}, v_{i}^{2} \in V_{i}$. Since $f\left(V_{i}\right) \subseteq V_{i}, f \mid V_{i}$ belongs to $C\left(T_{i} ; V_{i}\right)$. Using an argument almost 
identical to the proof of Theorem 1 of [5], it is seen that $f \mid V_{i}$ is linear since $n_{i}>1$.

Assume now that $C(R ; V)$ is a ring for each $R$-module $V$ but $R$ is not strongly noncommutative. Then in the decomposition $R=T_{1} \oplus \cdots \oplus T_{t}+M$ at least one $T_{i}$ is a local ring, say $T_{1}$. We know $R / \operatorname{rad} R \simeq K_{1} \oplus S_{2} \oplus \cdots \oplus S_{t}$ where $K_{1}$ is a field and each $S_{i}$ is a simple ring. Also under the homomorphism $R \rightarrow$ $R / \operatorname{rad} R, T_{1} \rightarrow K_{1}, T_{2} \rightarrow S_{2}, \ldots, T_{t} \rightarrow S_{t}$. Thus there exists a maximal ideal $I$ containing $T_{2}, T_{3}, \ldots, T_{t}$ and $\operatorname{rad} R$ such that $R / I \cong K_{1}$. Under the action $r \bar{k}=\overline{r k}, R / I$ is an irreducible $R$-module. Also $V=R \oplus R / I \oplus R / I$ is a faithful $R$-module under componentwise action. If we let $W=R / I \oplus R / I$ then $C(R ; W)$ can be embedded in $C(R ; V)$ as follows. For $\hat{g} \in C(R ; W)$, define $g: V \rightarrow V$ by $g\left(r+\bar{k}_{1}+\bar{k}_{2}\right)=\hat{g}\left(\bar{k}_{1}+\bar{k}_{2}\right)$. We note further that since $R / I$ is a field, $\operatorname{Ann}_{R}(W)$ $=I$ and so $C(R ; W) \cong C(R / I ; W) \cong C\left(K_{1} ; W\right)$. Since $\operatorname{dim}_{K_{1}} W=2$, it follows from Theorem 1 of [5] that $C\left(K_{1} ; W\right)$ and hence $C(R ; W)$ are not rings. Consequently $C(R ; V)$ is not a ring, a contradiction. Thus it must be the case that $R$ is strongly noncommutative.

For any finite ring $R$ there exists an $R$-module $V$ such that $C(R ; V)$ is a ring; e.g., let $V={ }_{R} R$. Moreover it is always the case that $\operatorname{End}_{R}(V) \subseteq C(R ; V)$. We now give an example to show that it is possible for $C(R ; V)$ to be a ring and yet $C(R ; V) \neq \operatorname{End}_{R}(V)$.

EXAMPLE 2.1. Let $R$ be the ring consisting of the $3 \times 3$ matrices of the form

$$
\left[\begin{array}{lll}
a & b & c \\
0 & a & 0 \\
0 & 0 & a
\end{array}\right], \quad a, b, c \in G F(2) .
$$

Let

$$
V=\left\{\left(\begin{array}{l}
x \\
y \\
z
\end{array}\right) \mid x, y, z \in G F(2)\right\} .
$$

A calculation shows that $\operatorname{End}_{R}(V)=R$. Another calculation gives $f(R v) \subseteq R v$ for each $f \in C(R ; V)$ and for each $v \in V$. From this it follows that $C(R ; V)$ is a ring since if $v \in V$ then

$$
f(g+h) v=f(g v+h v)=f\left(r_{1} v+r_{2} v\right)=\left(r_{1}+r_{2}\right) f(v)=(f g+f h) v .
$$

Let $\left\{e_{1}, e_{2}, e_{3}\right\}$ be the standard basis for the vector space $V$ over $G F(2)$. Then $V=R\left(e_{1}+e_{2}+e_{3}\right) \cup R e_{2} \cup R e_{3}$ and the relation $f\left(e_{1}+e_{2}+e_{3}\right)=f\left(e_{2}\right)=f\left(e_{3}\right)$ $=e_{1}$ determines a function in $C(R ; V)$. But $f$ is not in $\operatorname{End}_{R}(V)$ since $f\left(e_{2}+e_{3}\right) \neq$ $f\left(e_{2}\right)+f\left(e_{3}\right)$. Hence $\operatorname{End}_{R}(V) \neq C(R ; V)$.

In the next section we show that if $C(R ; V)$ is a semisimple ring then $C(R ; V)=$ $\operatorname{End}_{R}(V)$.

3. Semisimple centralizer near-rings. Let $C(R ; V)$ be semisimple. Then the center of $C(R ; V)$ cannot contain nonzero nilpotent elements. Hence the center of $R$ cannot contain nilpotent elements so the center of $R$ is a direct sum of fields. Thus if $n$ is the characteristic of $R$, we have $n=p_{1} p_{2} \cdots p_{s}$ where the $p_{i}$ 's are distinct primes. But this implies that $R=R_{1} \oplus \cdots \oplus R_{s}$ where $R_{i}$ has characteristic $p_{i}$. Because it has characteristic $p_{i}, R_{i}$ is an algebra over the field $G F\left(p_{i}\right)$ and so the 
Wedderburn principal theorem [7, p. 164] holds for $R_{i}$. Consequently $R=\Sigma_{i, j} \oplus S_{i j}$ $+N$ where each $S_{i j}$ is a simple ring and $N$ is a nilpotent ideal of $R$.

The following example shows that there exist semisimple centralizer near-rings that are not rings.

EXAMPLE 3.1. Let $R=\bar{R} \oplus F$ where $F=G F(2)$ and $\bar{R}$ is the simple ring of $2 \times 2$ matrices over $G F(2)$. Let $\left.V_{i}=\left\{\begin{array}{l}x \\ y\end{array}\right) \mid x, y \in F\right\}, i=1,2$, and let $R$ act on $V=V_{1} \oplus V_{2}$ componentwise. Then $C(R ; V) \cong C\left(\bar{R} ; V_{1}\right) \oplus C\left(F ; V_{2}\right)$ where $C\left(\bar{R} ; V_{1}\right)$ is a simple ring while $C\left(F ; V_{2}\right)$ is a simple near-ring which is not a ring. Hence $C(R ; V)$ is semisimple and not a ring.

For the remainder of this section we assume $C(R ; V)$ is semisimple and investigate when $C(R ; V)$ equals $\operatorname{End}_{R}(V)$. As we have seen $R=S_{1} \oplus \cdots \oplus S_{t}+N$ where each $S_{i}$ is simple and $N$ is a nilpotent ideal of $R$. We may assume $N \neq(0)$; otherwise $R$ is semisimple and the results of $\S 1$ apply.

Assume $t=1$, i.e. $R=S_{1}+N$. From the proof of Lemma 1 of [5] it follows that $C(R ; V)$ contains a function $f$ such that $g_{1} f g_{2} f=0$ for all $g_{1}, g_{2} \in C(R ; V)$. Hence $C(R ; V)$ contains a nilpotent $C(R ; V)$-subgroup and is not semisimple. So we may assume $t>1$.

Let $e_{i}$ denote the identity for $S_{i}$. Then $V=V_{1} \oplus \cdots \oplus V_{t}$ where $V_{i}=\{v \in$ $\left.V \mid e_{i} v=v\right\}$. Also for $i, j=1,2, \ldots, t$ let $N_{i j}=e_{i} N e_{j}$. Then $N=\sum N_{i j}$. For $i=$ $1, \ldots, t$ let $B_{i}=\left\{w_{i} \in V_{i} \mid w_{i}=n_{i j} v_{j}\right.$ for some $\left.j \neq i, n_{i j} \in N_{i j}, v_{j} \in V_{j}\right\}$, and let $W$ denote the subgroup of $V$ generated by $B_{1} \cup B_{2} \cup \cdots \cup B_{t}$. Finally let $W_{L}=$ $\{w \in V \mid f(w+v)=f(w)+f(v)$ for all $v \in V, f \in C(R ; V)\}$.

LEMMA 3.1. $W$ is an $R$-submodule of $V, W_{L}$ is a subgroup of $V$ and $W \subseteq W_{L}$.

Proof. An element of $W$ has the form $w=\sum n_{i j} v_{j}$ with $i \neq j$. For $n_{k l} \in N_{k l}$ and $n_{i j} v_{j} \in B_{j}$ we have $n_{k l} n_{i j} v_{j} \in B_{k}$ if $k \neq j$ and $n_{k l} n_{i j} v_{j}=n_{k l}\left(n_{i j} v_{j}\right) \in B_{k}$ if $k=j$. In this manner it is seen that $N W \subseteq W$. Also if $s \in S_{1} \oplus \cdots \oplus S_{t}$ then $s n_{i j} v_{j}=$ $\left(s n_{i j}\right) v_{j} \in B_{i}$ since $s n_{i j} \in N_{i j}$. Hence $S W \subseteq W$ and $W$ is an $R$-submodule of $V$.

The second part of the lemma is straightforward and is omitted. To prove the last part if suffices to show that $B_{i} \subseteq W_{L}$ for each $i$. To this end let $v_{i}=n_{i j} v_{j} \in B_{i}$, $f \in C(R ; V)$. For $k \neq i$ we have $f\left(v_{i}+v_{k}\right)=f\left(v_{i}\right)+f\left(v_{k}\right)$. For $v_{i}^{\prime} \in V_{i}$,

$$
\begin{aligned}
f\left(v_{i}+v_{i}^{\prime}\right) & =f\left(n_{i j} v_{j}+v_{i}^{\prime}\right)=f\left(\left(n_{i j}+e_{j}\right)\left(v_{j}+v_{i}^{\prime}\right)\right) \\
& =\left(n_{i j}+e_{j}\right) f\left(v_{j}+v_{i}^{\prime}\right)=\left(n_{i j}+e_{j}\right)\left[f\left(v_{j}\right)+f\left(v_{i}\right)\right]=f\left(v_{i}\right)+f\left(v_{i}^{\prime}\right) .
\end{aligned}
$$

With this it is easy to see that $f\left(v_{i}+v\right)=f\left(v_{i}\right)+f(v)$ for all $v \in V$, as desired.

From the lemma, every $f \in C(R ; V)$ is linear on $W$ and moreover $f(W) \subseteq W$. Suppose now that $C(R ; V)$ is simple. Then the map $f \rightarrow f \mid W$ is an imbedding of $C(R ; V)$ into $\operatorname{End}_{R}(W)$. Also $W \neq(0)$, for otherwise $N_{i j} V_{j}=(0)$ for each $i \neq j$ and so each $V_{i}$ is an $R$-module and $C(R ; V)$-invariant. Hence $C(R ; V)$ would not be simple. Thus $W \neq 0$ and $C(R ; V)$ is a ring. This provides an alternate proof to Theorem 2 of [5].

LEMMA 3.2. If the simple ring $S_{i}$ is not a field then every $f \in C(R ; V)$ is linear on $V_{i}$ 
Proof. Again the restriction map $f \rightarrow f \mid V_{i}$ is a homomorphism of $C(R ; V)$ into $C\left(S_{i} ; V_{i}\right)$. Since $C\left(S_{i} ; V_{i}\right)=\operatorname{End}_{S_{i}}\left(V_{i}\right)$, every $f \in C(R ; V)$ is linear on $V_{i}$.

Let $v_{i}$ be a nonzero element in $V_{i}$. Then from the chain of $S_{i}$-submodules of $V_{i}$,

$$
\text { (0) } \subseteq \operatorname{ker} N \cap V_{i} \subseteq \operatorname{ker} N^{2} \cap V_{i} \subseteq \cdots \subseteq \operatorname{ker} N^{k-1} \cap V_{i} \subseteq V_{i},
$$

we see that there exists a unique integer $\rho\left(v_{i}\right)$ such that $v_{i} \in \operatorname{ker} N^{\rho\left(v_{i}\right)} \cap V_{i}$ but $v_{i} \notin \operatorname{ker} N^{\rho\left(v_{i}\right)-1} \cap V_{i}$. We call $\rho\left(v_{i}\right)$ the rank of $v_{i}$. For completeness let 0 have rank 0 . We note that for $v_{i}, v_{i}^{\prime}$ in $V_{i}$ we have $\rho\left(v_{i}+v_{i}^{\prime}\right) \leqslant \max \left\{\rho\left(v_{i}\right), \rho\left(v_{i}^{\prime}\right)\right\}$.

Lemma 3.3. If $\operatorname{ker} N \cap V_{i}=\{0\}$ then every $f \in C(R ; V)$ is linear on $V_{i}$.

Proof. Assume $f \in C(R ; V)$ such that $f$ is not linear on $V_{i}$. Then there exist $v_{i}$, $v_{i}^{\prime}$ in $V_{i}$ such that $f\left(v_{i}+v_{i}^{\prime}\right)-f\left(v_{i}\right)-f\left(v_{i}^{\prime}\right) \neq 0$. Among all such pairs $\left\{v_{i}, v_{i}^{\prime}\right\}$ select one pair having an element of minimal rank, say $\left\{x_{i}, x_{i}^{\prime}\right\}$, where $x_{i}$ has minimal rank. For each $n_{j i} \in N_{j i}$ where $j \neq i$ we have $n_{j i}\left(f\left(x_{i}+x_{i}^{\prime}\right)-f\left(x_{i}\right)-f\left(x_{i}^{\prime}\right)\right)=0$, since $n_{j i} x_{i} \in W$. Due to the minimality of $x_{i}$ we also have

$$
n_{i i}\left(f\left(x_{i}+x_{i}^{\prime}\right)-f\left(x_{i}\right)-f\left(x_{i}^{\prime}\right)\right)=0
$$

for each $n_{i i} \in N_{i i}$. Hence $f\left(x_{i}+x_{i}^{\prime}\right)-f\left(x_{i}\right)-f\left(x_{i}^{\prime}\right) \in \operatorname{ker} N \cap V_{i}$, a contradiction.

TheOREM 3.1. Let $C(R ; V)$ be a semisimple near-ring where $R$ is not semisimple. Then $R=S_{1} \oplus \cdots \oplus S_{t}+N$ where $t>1$, each $S_{i}$ is a simple ring and $N$ is $a$ nonzero nilpotent ideal of $R$. Moreover the following are equivalent.

(i) $C(R ; V)$ is a ring.

(ii) $C(R ; V)=\operatorname{End}_{R}(V)$.

(iii) For each $i$ at least one of the following is true:

(a) $S_{i}$ is not a field;

(b) $S_{i}$ is a field and $\operatorname{dim}_{S_{i}}\left[\operatorname{ker} N \cap V_{i}\right] \leqslant 1$;

(c) $V_{i} \subseteq W$.

Proof. The first part of the theorem has already been established. For the equivalences we start with (iii) $\rightarrow$ (ii). From Lemma 3.2 if $S_{i}$ is not a field then every $f \in C(R ; V)$ is linear on $V_{i}$. The same conclusion is true if $V_{i} \subseteq W$. So we may assume that at least one $S_{i}$ is a field, say $S_{1}$, with $\operatorname{dim}_{S_{1}}\left[\operatorname{ker} N \cap V_{1}\right]<1$ and $V_{1} \& W$. If $\operatorname{ker} N \cap V_{1}=(0)$ then Lemma 3.3 applies. Therefore, we may also assume ker $N \cap V_{1}$ is a 1-dimensional vector space over $S_{1}$.

Let $W_{1}=W \cap V_{1}$ and $S=S_{1} \oplus \cdots \oplus S_{t}$. $V$ is a completely reducible $S$-module and we have, as $S$-modules, $V=\bar{V}_{1} \oplus W_{1} \oplus X$ where $X=V_{2} \oplus \cdots \oplus V_{t}$, and $V_{1}=\bar{V}_{1} \oplus W_{1}$. Note that $W_{1} \oplus X$ is an $R$-module and is $C(R ; V)$-invariant. We select an $S_{1}$-basis $\left\{v_{1}, v_{2}, \ldots, v_{l}, w_{1}, \ldots, w_{m}\right\}$ for $\bar{V}_{1} \oplus W_{1}$ as follows. Let $\left\{w_{1}, \ldots, w_{m}\right\}$ be a basis for $W_{1}$. Let $\left\{v_{t_{1}}, \ldots, v_{l}\right\}$ be a basis for $\bar{V}_{1}^{1}=\{v \in$ $\left.\bar{V}_{1} \mid N_{11} v \subseteq W_{1}\right\}$. Let $\left\{v_{t_{2}}, \ldots, v_{t_{1}}, \ldots, v_{l}\right\}$ be a basis for $\bar{V}_{1}^{2}=\left\{v \in \bar{V}_{1} \mid N_{11} v \subseteq\right.$ $\left.\bar{V}_{1}^{1}\right\}$, etc. Using the fact that $N_{11}$ is nilpotent, this process terminates to give the desired basis $\left\{v_{1}, \ldots, v_{l}, w_{1}, \ldots, w_{m}\right\}$ for $\bar{V}_{1} \oplus W_{1}$. Thus every $v \in V$ can be uniquely represented in the form $v=s_{11} v_{1}+\cdots+s_{1 l} v_{l}+w+x$ where $s_{1 i} \in S_{1}$, $w \in W_{1}, x \in X$.

Let $k$ be a nonzero element in ker $N \cap V_{1}$. The function $f: V \rightarrow V$ defined by 
$f\left(s_{11} v_{1}+\cdots+s_{11} v_{l}+w+x\right)=s_{11} k$ belongs to $C(R ; V)$. Let $L=C(R ; V) f$, the $C(R ; V)$-subgroup generated by $f$. If $k \in W_{1}$ then $g(k) \in \operatorname{ker} N \cap W_{1}$ for each $g \in C(R ; V)$, and thus $g_{1} f g_{2} f=0$. Thus $L^{2}=(0)$, a contradiction to $C(R ; V)$ being semisimple. Hence ker $N \cap W_{1}=(0)$ and, since $\bar{V}_{1}$ was an arbitrary complement of $W_{1}$ in $V_{1}$, we may reselect $\bar{V}_{1}$ if necessary such that ker $N \cap V_{1} \subseteq \bar{V}_{1}$; i.e. $V_{1}=\tilde{V}_{1} \oplus\left(\operatorname{ker} N \cap V_{1}\right) \oplus W_{1}$ where $\bar{V}_{1}=\tilde{V}_{1} \oplus(\operatorname{ker} N$ $\cap V_{1}$ ). If $\tilde{V}_{1} \neq(0)$ then we may assume our first basis element $v_{1}$ belongs to $\tilde{V}_{1}$. But once again, if $f$ is defined as above, we get $L^{2}=(0)$. Hence $\tilde{V}_{1}=(0)$ and $\bar{V}_{1}=\operatorname{ker} N \cap V_{1}$. We now have $V=\left(\operatorname{ker} N \cap V_{1}\right) \oplus W_{1} \oplus X$. Since

$$
\operatorname{dim}_{S_{1}}\left(\operatorname{ker} N \cap V_{1}\right)=1 \text {, }
$$

each $f \in C(R ; V)$ is trivially linear on ker $N \cap V_{1}$ and hence on all of $V_{1}$. This shows that (iii) $\rightarrow$ (ii).

Suppose (i) is true. Then we may assume by way of contradiction that some $S_{i}$ is a field, say $S_{1}$, that $\operatorname{dim}_{S_{1}}\left[\operatorname{ker} N \cap V_{1}\right]>1$ and that $V_{1} \& W$. Because $C(R ; V)$ is semisimple the arguments above imply $V=\left(\operatorname{ker} N \cap V_{1}\right) \oplus W_{1} \oplus X$ where $W_{1}$ and $X$ are defined as before. But ker $N \cap V_{1}$ and $W_{1} \oplus X$ are both $R$-modules and both $C(R ; V)$-invariant. Hence

$$
C(R ; V) \cong C\left(S_{1} ; \operatorname{ker} N \cap V_{1}\right) \oplus C\left(R ; W_{1} \oplus X\right) .
$$

Since $\operatorname{dim}_{S_{1}}\left(\operatorname{ker} N \cap V_{1}\right)>1$, the first summand is not a ring. Hence (i) $\rightarrow$ (iii). Since (ii) $\rightarrow$ (i) is obvious the proof is complete.

As a consequence of this theorem we note that if $C(R ; V)$ is a simple ring where $R$ is not a field then $C(R ; V)=\operatorname{End}_{R}(V)$. This was stated as Theorem 3 in [5] but the proof given there is incorrect.

We also note that as a consequence of the proof of Theorem 3.1 and the preliminaries to it we have the following structural result for semisimple near-rings of the form $C(R ; V)$.

Corollary. If $C(R ; V)$ is semisimple then $C(R ; V)=A_{1} \oplus \cdots \oplus A_{\text {t }}$ where each $A_{i}$ is either a simple ring or a simple near-ring of the form $C\left(F_{i} ; V_{i}\right)$ where $V_{i}$ is a vector space over a field $F_{i}$. Moreover if $R$ is not semisimple then at least one $A_{i}$ must be a ring.

Proof. It remains to prove the last part of the corollary. Since $C(R ; V)$ is semisimple then $R=S_{1} \oplus \cdots \oplus S_{k}+N$ where $N=\operatorname{rad} R$ and each $S_{i}$ is simple with identity $e_{i}$. As before let $N_{i j}=e_{i} N e_{j}$ and let $W$ be the $R$-submodule of $V$ as in Lemma 3.1. If $W=(0)$ then $N_{i j} V_{j}=(0)$ for each $i \neq j$ where $V_{j}$ is the 1-space for $e_{j}$. This means each $V_{i}$ is an $R$-module as well as $C(R ; V)$-invariant. Hence

$$
C(R ; V) \simeq C\left(R_{1} ; V_{1}\right) \oplus \cdots \oplus C\left(R_{k} ; V_{k}\right)
$$

where $R_{i}=S_{i}+N_{i i}$. Since $C(R ; V)$ is semisimple each $C\left(R_{i} ; V_{i}\right)$ is semisimple [8, p. 146]. We show now that if $N_{i i} \neq(0)$ then $C\left(R_{i} ; V_{i}\right)$ cannot be semisimple. Suppose $N_{i i}^{l}=(0)$ but $N_{i i}^{l-1} \neq(0)$. Let $W_{1}=\operatorname{ker} N_{i i}^{l-1}=\left\{v \in V_{i} \mid n v=0\right.$ for all $\left.n \in N_{i i}^{l-1}\right\}$, a proper subgroup of $V_{i}$, an $S_{i}$-submodule, and $C\left(R_{i} ; V_{i}\right)$-invariant. As an $S_{i}$-module $V_{i}$ is completely reducible so $V_{i}=W_{1} \oplus W_{2}$, an $S_{i}$-module direct 
sum. As constructed in the proof of Lemma 1 of [5] there exists a nonzero $f \in C\left(R_{i} ; V_{i}\right)$ such that $f\left(V_{i}\right) \subseteq W_{1}$ and $f\left(W_{1}\right)=\{0\}$. Let $I=\{f \in$ $C\left(R_{i} ; V_{i}\right) \mid f\left(V_{i}\right) \subseteq W_{1}$ and $\left.f\left(W_{1}\right)=\{0\}\right\}$. Then $I$ is a nilpotent $C\left(R_{i} ; V_{i}\right)$-subgroup $\left(I^{2}=(0)\right)$ and hence $C\left(R_{i} ; V_{i}\right)$ is not semisimple. So each $N_{i i}=(0)$ and since $N_{i j} V=(0), N_{i j}=(0)$ if $i \neq j$. Thus $R$ is semisimple.

So we may assume $W \neq(0)$. Since $W$ is $C(R ; V)$-invariant the $\operatorname{map} f \rightarrow f \mid W$ is a homomorphism of $C(R ; V)$ into the ring $\operatorname{End}_{R}(W)$. Hence a nontrivial homomorphic image of $C(R ; V)$ is a ring and this implies at least one simple component of $C(R ; V)$ is a ring $[8$, p. 55].

\section{REFERENCES}

1. G. Betsch, Some structure theorems on 2-primitive near-rings, Rings, Modules and Radicals (Proc. Colloq., Keszthely, 1971), Colloq. Math. Soc. János Bolyai, Vol. 6, North-Holland, Amsterdam, 1973, pp. 73-102.

2. C. J. Maxson and K. C. Smith, The centralizer of a group automorphism, J. Algebra 54 (1978), $27-41$.

3.

4. __ The centralizer of a set of group automorphisms, Comm. Algebra 8 (1980), 211-230.

5. __ Simple near-ring centralizers of finite rings, Proc. Amer. Math. Soc. 75 (1979), 8-12.

6.__ Near-ring centralizers, Proc. Ninth Annual USL Math. Conf. Research Series 48, Univ. Southwestern Louisiana, April, 1979, pp. 49-58.

7. B. R. McDonald, Finite rings with identity, Dekker, New York, 1974.

8. G. Pilz, Near-rings, The Theory and its Applications, North-Holland Math. Studies, No. 23, North-Holland, Amsterdam, New York and Oxford, 1977.

Department of Mathematics, Texas adM Universtty, College Station, Texas 77843 